\title{
Interview mit ASP-Mitglied Esther Bulang
}

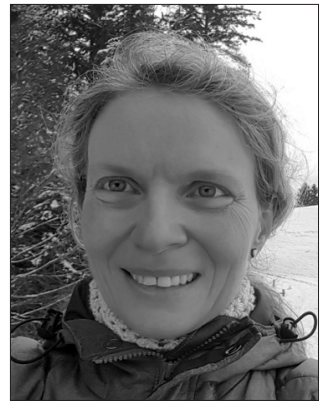

Esther Bulang
Was waren Ihre Beweggründe, den Beruf einer Psychotherapeutin zu wählen?

Ich wollte den Menschen in seiner Ganzheitlichkeit - die Arbeit am und mit dem Menschen und eine ganzheitliche Perspektive - in den Mittelpunkt meiner beruflichen Tätigkeit stellen. Und ich wollte wirklich Zeit haben für Menschen, wissen, was sie beschäftigt, wie sie denken, was sie fühlen, was sie wollen. Ich habe sehr viel darüber nachgedacht, was mir wirklich wichtig ist im Leben und was meiner Persönlichkeit am meisten entspricht, welche Arbeit und wie ich sie machen möchte, was mich glücklich machen würde und womit ich mich am besten einbringen kann. Psychotherapeutin sein zu können ist für mich, eine Lebensphilosophie zu leben.

Was ist Ihr beruflicher Hintergrund/Werdegang und welche Bedeutung hat dieser für Ihre berufliche Tätigkeit?

Unmittelbar nach dem Abitur war es in der ehemaligen DDR Pflicht, vor dem Medizinstudium ein Jahr als pflegerische Hilfskraft in einem Krankenhaus zu arbeiten. Danach folgte mein Medizinstudium in Leipzig, das genau im Jahr des Mauerfalls 1989 endete. Noch heute verbindet der Umbruch, der damals in Leipzig eingeleitet wurde, mich tief mit diesem Ort. Die während des Studiums obligatorische Diplomarbeit, in meinem Fall von Art und Umfang eine wissenschaftliche Arbeit, wurde aus politischen Gründen nicht als Promotionsarbeit anerkannt. Nach dem Studium, inzwischen Mutter zweier wunderbarer Kinder, absolvierte ich eine fünfjährige Ausbildung zur Fachärztin für Augenheilkunde und arbeitete fortan als solche im klinischen Bereich. 2003 schloss ich dann eine Promotion im Fachbereich der Neurologie/Neuroophthalmologie an der TU Dresden ab. Der Abschluss der Promotion war begleitet von Vortragsreisen, auch in den USA, eine enorm wichtige Erfahrung.

Als 2006 mein Entschluss fiel, nun ganz und gar Psychotherapeutin werden zu wollen, war ich auf dem Höhepunkt meines Erfolgs als Leiterin einer kleineren Augenklinik und als Operateurin in der intraokularen Mikrochirurgie angekommen. Zu diesem Zeitpunkt hatte ich ca. elf Jahre fast täglich Augen operiert. Mein Erfolg beruhte neben dem operativen Erfolg hauptsächlich darauf, dass ich mein Gefühl für den Menschen, den ich operierte, und für mein ganzes Team in die strukturierte und rationale Tätigkeit integrieren lernte, mich sehr zugewandt und individuell um die Menschen kümmerte. Etwas, wofür insbesondere ältere Menschen, die Hilfe benötigen, sehr dankbar sind. Die älteste Patientin, die ich operierte, war 97 Jahre alt. Ich merkte aber auch, dass lange OP-Tage mit einförmiger körperlicher Haltung am OP-Mikroskop mir zunehmend körperliche Beschwerden bereiteten und war auf der Suche nach einer Vision, wie ich mit guter Lebensqualität meine Berufsjahre bis zur Pensionierung gestalten wollte. Da weder der stationäre Bereich mit seiner zunehmenden Ökonomisierung noch der ambulante Bereich mit hoher Arbeitsdichte und wenig Zeit für den Patienten mir eine zufriedenstellende Arbeitssituation boten, war irgendwie ein Endpunkt meiner beruflichen Tätigkeit erreicht. Bis hierhin hatte ich Menschen auf der körperlichen Ebene zu einem besseren Sehen geholfen, nun sollte es darum gehen, die Seele des Menschen sehender zu machen. Circa zwei Jahre parallel berufsbegleitend zu meiner Tätigkeit begann ich meine Ausbildung am Dresdner Institut für Psychodynamische Psychotherapie e.V. mit regelmässiger Teilnahme an Abendseminaren und dem Beginn einer Lehranalyse. Zuvor als Oberärztin vor der Übernahme der Leitung der Klinik erhielt ich von der Geschäftsleitung des Klinikums für einen Monat eine unbezahlte Freistellung und arbeitete als operierende Augenärztin und Trainerin für eine neue OP-Methode in einer Augenklinik in Kenia ausserhalb von Nairobi, darüber hinaus auch in einem Kurzeinsatz als flying doctor in einem Buschhospital. $\mathrm{Zu}$ den prägendsten Erfahrungen aus dieser Arbeit gehören u. a. der Respekt vor dem Anderssein und die Akzeptanz, niemanden retten zu können, die Grenzen meiner Möglichkeiten bezogen auf das Leben und die Gesundheit anderer zu respektieren. Den Übergang von meinem Leben als Augenärztin zur Psychotherapeutin feierte ich mit einer vierwöchigen Reise, Hochgebirgstrekking zu Fuss von Nepal nach Tibet und in Tibet die Umrundung, Kora, des heiligen Berges Kailash und der Besuch von Lhasa. Eine zutiefst spirituelle Erfahrung. Ich habe dann in Deutschland den zweiten Facharztabschluss in «Psychosomatischer Medizin und Psychotherapie» gemacht. Da für mich von Beginn des Wechsels in die Psychotherapietätigkeit an klar war, dass ich Körperpsychotherapeutin werden wollte, führte mich der Mangel 
an Ausbildungsmöglichkeiten in Deutschland in die Schweiz. Mein damaliger Klinikchef hat diese Ausbildung aktiv unterstützt. In der Schweiz habe ich über sieben Jahre berufsbegleitend parallel zur tiefenpsychologisch-psychodynamischen psychoanalytischen Ausbildung in Deutschland eine sehr intensive Körperpsychotherapieausbildung am Institut für Biosynthese in Heiden bei David und Silvia Boadella absolviert.

Danach habe ich über eineinhalb Jahre eine Ausbildung zur Therapeutin für Therapeutisches Bogenschiessen gemacht, die unter anderem beinhaltet zu reflektieren, mit einer Waffe umzugehen, und auch sehr bewusst weibliche und männliche Energien in mir wahrzunehmen. Seit 2014 bin ich in der Aus- und Fortbildung für Traumatherapie mit der Spezialisierung für chronische Traumatisierung und dissoziative Störungen bei Ellert Nijenhuis. In der Biosynthese und Traumatherapie nach Nijenhuis war auch eine Ausbildung zur Supervisorin eingeschlossen. Beides darf ich auch unterrichten. Ein ganz persönliches Interesse und einen langen Wunschtraum erfülle ich mir, indem ich mich in komplementären Therapieformen weiterbilde und mich dem Thema Spiritualität im Leben und in der Psychotherapie widme. Seit neun Jahren absolviere ich eine Selbsterfahrung und Training in Schamanismus bei der koreanischen Schamanin Hiah Park und bei dem grönländischen Schamanen Angaangaq Angakkorsuaq sowie seit mehr als 13 Jahren eine umfangreiche Ausbildung in tibetischem Buddhismus und tibetisch buddhistischen Behandlungstechni- ken. Ich habe eine Ausbildung in energetischer Massage Kum Nye und buddhistischer Psychologie abgeschlossen, bin Lehrerin für Tibetisches Heilyoga Lu Jong und beende dieses Jahr meine Ausbildung zur tibetisch buddhistischen Meditationslehrerin bei Lharampa Tenzin Kalden. Neben der Lehranalyse, der Körperpsychotherapie- und der Traumatherapieausbildung ist für mich die schamanische Ausbildung die tiefste Selbsterfahrung. Auch deshalb, weil ich einer kleinen nationalen westslawischen Minderheit, den Sorben-Wenden, angehöre, die in Ostdeutschland in Nachbarschaft zu Polen und Tschechien leben. Diese Identitätsfindung beinhaltet auch die Suche nach meinen Wurzeln vor der Christianisierung und Assimilierung in die deutsche Kultur. Meine Arbeitstätigkeit beträgt nur 50-70 Prozent. Damit verzichte ich ganz bewusst auf Dinge des Alltagslebens, die ich mir früher finanziell leisten konnte, lebe bescheidener und bewusster. Ich könnte nicht glücklich sein, wenn neben hundertprozentiger Berufstätigkeit keine Zeit mehr für das Engagement in gesellschaftlichen Bereichen wie Klimaschutz und Umwelt übrigbliebe.

Arbeiten Sie als selbstständige Psychotherapeutin in freier Praxis und/oder sind Sie zusätzlich noch als delegierte Psychotherapeutin tätig?

Bedingt durch meine Ausbildungen und Abschlüsse in Deutschland und der Schweiz arbeite ich angestellt in einer ärztlichen Praxis hauptsächlich als selbstständige Psychotherapeutin mit der Anerkennung der SAPPM und darf durch die Anerkennung der FMPP (zwei Fähig-

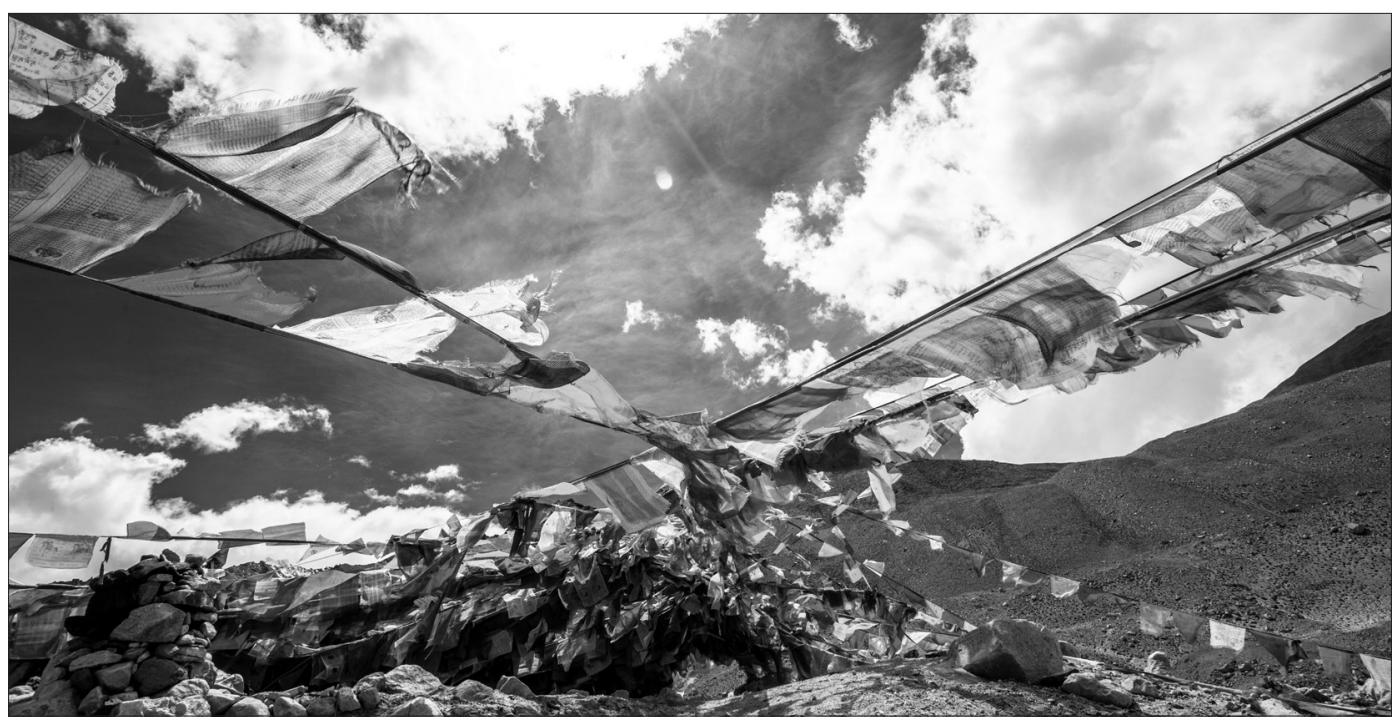


keitsausweise für Ärzte in der Schweiz) auch Psychotherapie delegieren. Weiterhin habe ich eine eigene Praxis, in der ich als selbstständige Psychotherapeutin tätig bin.

Gibt es noch einen weiteren Beruf, eine weitere Beschäftigung, den/die Sie zusätzlich zur Psychotherapie ausüben?

In der ärztlichen Praxis arbeite ich noch in einem geringen Umfang assistenzärztlich in der Komplementärmedizin (orthomolekulare Medizin).

In meiner eigenen Praxis möchte ich demnächst Tibetisches Heilyoga Lu Jong, tibetische Massage Kum Nye, tibetisch buddhistische Meditation und, wenn möglich, therapeutisches Bogenschiessen sowie energetische Heilarbeit anbieten. Das realisieren zu können, hat vor allem einen finanziellen Aspekt, also geht es langsam und entsprechend den gerade gegebenen Möglichkeiten. Eine Website befindet sich aktuell im Aufbau.

\section{Was sind hierfür die Beweggründe?}

Bezüglich des Heilyoga und der Meditation einerseits tue ich es für mich, es ist ein schöner Ausgleich. Und gleichermassen ist es Ausdruck meiner ganzheitlichen Philosophie für den Menschen und Patienten. Alles kann man nicht in eine Psychotherapiestunde integrieren, und, es gibt so viele schöne Dinge, die uns helfen können seelisch und körperlich gesund zu bleiben oder zu werden und zufrieden leben zu können.

Was ist Ihre Spezialisierung?

Meine Spezialisierungen sind die Traumatherapie, insbesondere für chronische Traumatisierung und dissoziative Störungen (Enaktive Traumatherapie nach Nijenhuis), Körperpsychotherapie Biosynthese nach David und Silvia Boadella sowie veränderte Bewusstseinszustände und Spiritualität. Weiterhin in Verbindung mit meiner komplementärtherapeutischen medizinischen Tätigkeit die Behandlung von Burnout.

Fühlen Sie sich mit Ihrer beruflichen Situation zufrieden?

Im Grossen und Ganzen fühle ich mich sehr zufrieden mit meiner Situation, weil ich eine sogennnte «Nische» für meine psychotherapeutische Tätigkeit gefunden habe, in der ich mich in einem angestellten Verhältnis fair behandelt fühle und ganz selbstständig meine psychotherapeutische Tätigkeit im Rahmen der Grundver- sicherung ausüben kann. Das heisst, ich kann selbstständig die Indikation zur Psychotherapie stellen und ohne aufwändige Formalitäten die psychotherapeutische Unterstützung geben, die Menschen in diesem Augenblick benötigen. Dies ist mir durch meine Doppelanerkennung als Ärztin und Psychotherapeutin möglich. Ich bin sehr dankbar, dass das Schweizer System dies ermöglicht. Gleichermassen bin ich mir auch bewusst, dass dies nur in dieser «Nische» für mich so möglich ist. Über die Suche nach diesem Platz bin ich sehr mit den Problemen der psychologischen Psychotherapeuten und -therapeutinnen in einem Anstellungsverhältnis bei einem Psychiater oder einer Psychiaterin konfrontiert worden. Wenn es sicher auch gute Arbeitsbeziehungen zwischen PsychiaterInnen und psychologischen PsychotherapeutInnen gibt, ist doch der Eindruck hängen geblieben, dass es psychologischen Psychotherapeuten und Psychotherapeutinnen oftmals nicht so gut geht in diesem Abhängigkeitsverhältnis, das ich in dem gehörten Ausmass nicht akzeptieren könnte.

Ich weiss nicht, ob ich als selbstständige Psychotherapeutin in meiner freien Praxis im Grossraum Zürich aktuell genug für meinen Lebensunterhalt verdienen könnte. Und ich fühle mich nicht gut dabei, dass ich mit meiner Qualifikation als selbstständige Psychotherapeutin Menschen nicht im gleichen Umfang helfen kann, etwa wenn die finanzielle Unterstützung der Zusatzversicherungen bald aufgebraucht ist und es Menschen gibt, die dringend Psychotherapie bräuchten, sie aber nicht aus eigener Tasche weiter bezahlen können, weil die Grundversicherung die selbstständige Tätigkeit von PsychotherapeutInnen nicht finanziert.

Ich freue mich sehr, dass das Schweizer System mit der Durchsetzung des Psychologieberufegesetzes nicht die Vielfalt der Psychotherapie grundsätzlich eingeschränkt hat, wie das in Deutschland der Fall ist. Und meine grosse Anerkennung und mein Dank gelten neben dem BAG dem PsychotherapeutInnen-Verband ASP, sich dafür stark gemacht und gewirkt zu haben.

Gibt es etwas, dass Sie sich anders wünschen? Ich wünsche mir, dass, nachdem mit dem Psychologieberufegesetz einheitliche Massstäbe für die Ausbildung von psychologischen Psychotherapeuten und -therapeutinnen geschaffen wurden, diese als eigenständige Berufsgruppe selbst- 
ständig für die Grundversicherung tätig werden können. Wenn das System sich selbst und seiner Arbeit vertraut und jede anerkannte Psychotherapieschule sich der Verantwortung ihrer Ausbildung voll bewusst ist und dieser auch nachkommt, sollte dies absolut möglich sein.

Ich wünsche mir auch, dass viel mehr Akzeptanz für Teilzeitanstellungen besteht und damit viel mehr Möglichkeiten für die Menschen geschaffen werden, die so arbeiten möchten.

Ich wünsche mir ausserdem, dass es mehr unvoreingenommene Offenheit gepaart mit Neugierde, Akzeptanz und Respekt gegenüber komplementären Methoden gibt, was insbesondere auch bedeuten würde, Spiritualität als aktiven Wirkfaktor in der Psychotherapie anzuerkennen. Ich wünsche mir, dass sich der Bereich Psychotherapie als eigenständiger Bereich mehr für Nachbardisziplinen öffnet und Vernetzungen, z.B. mit der Philosophie, Kultur- oder Naturwissenschaft wie der Quantenphysik, entstehen, aus denen die Psychotherapie allgemeingültige Prinzipien des Daseins, Denkens und Handelns ableiten und daraus ein gesellschaftliches Engagement entstehen kann.

Gibt es etwas, das Sie sich von Ihrem Verband ASP wünschen?

Ich würde mir wünschen, dass die ASP sich stärker im gesellschaftlichen und politischen Bereich engagiert, z. B. bei Gesetzesvernehmlassungen oder mit Aufklärungsarbeit oder Stellungnahmen. Zwei ganz wichtige Bereiche wären die Schulbildung und pädagogische Psychologie. Auch wenn Psychotherapie per se bewertungsfrei ist, bin ich der Meinung, dass sie mit ihrem Wissen eine Verantwortung in der Gesellschaft wahrzunehmen hat.

Ich habe mich z. B. sehr gefreut, dass der Verband mit der Publikation der Aktion auf seiner Website die Initiative und Unterschriftensammlung «Referendum gegen die Überwachung von Versicherten» unterstützt hat. So etwas würde ich mir öfter und mehr wünschen, auch eine aktive Stellungnahme in den öffentlichen Medien und vielleicht auch eine Initiative selbst ins Leben zu rufen.

Ich wünsche mir, dass der Verband weiterhin den Weg der Anerkennung der Methodenvielfalt unterstützt und ihn weiterhin geht. Dieser Weg zeichnet für mich die Arbeit des Verbandes über all die Jahre seines Wirkens am meisten aus.

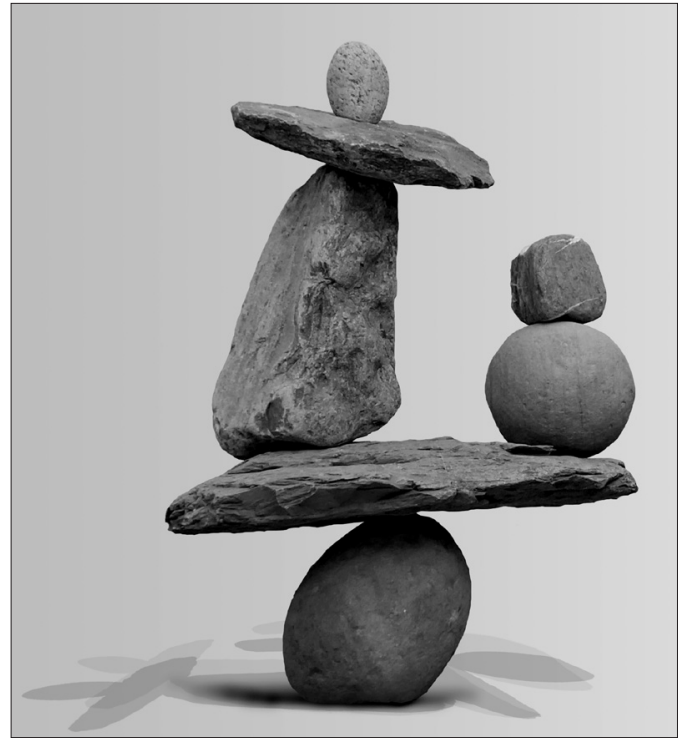

Fühlen Sie sich in Ihrem Berufsverband ASP vertreten und gewürdigt?

Ich fühle mich von meinem Berufsverband ASP vertreten und gewürdigt. Ich bin sehr dankbar für die Unterstützung, die ich seitens des Verbandes erfahren habe, als ich in die Schweiz kam und es um die Anerkennung meiner Ausbildung in Deutschland und der Schweiz ging. Im Gegensatz zu vielen anderen Stellen, mit denen ich Kontakt hatte, empfand ich den mit den einzelnen Personen der ASP als unkompliziert, unterstützend und offen. Das hat mich damals sehr beeindruckt und unterstützt. Auch, dass es einen Berufsverband gibt, der Körperpsychotherapie als Behandlungsmethode der Psychotherapie anerkennt.

Über die Befürwortung des Anordnungsmodells bin ich nicht glücklich, auch wenn ich die Beweggründe des Verbandes nachvollziehen kann. Ich bin der Meinung, Psychotherapeuten und Psychotherapeutinnen verfügen über so gute Kompetenzen, dass sie selbstständig als Berufsstand anerkannt werden und somit auch wirken sollen. Und dass das jetzt, wo Veränderungen auf diesem Gebiet passieren, gemacht werden muss. Nach meinem Dafürhalten wird das Anstossen einer erneuten Veränderung wieder viel Kraft und Zeit kosten, wenn erst einmal das Anordnungskonzept etabliert ist.

Ich finde, der Verband leistet mit der Realisierung des methodenübergreifenden Konzepts der Psychotherapieweiterbildung ASP Integral eine grossartige Arbeit und möchte mich dafür 
vielmals bedanken, auch wenn ich von meiner Ausbildung in der Biosynthese her davon nicht profitieren kann. Ich bin jedoch glücklich, dass anderen kleineren Ausbildungsschulen auf diese Weise geholfen werden kann, ihre Anerkennung zu erhalten und sie somit bestehen bleiben können.

Was wäre Ihr Fokus, wenn Sie im Vorstand der ASP wären?

Ganz klar die Öffentlichkeitsarbeit im politischen und rechtlichen Bereich und Aufklärungsarbeit in der Bevölkerung. Wahrscheinlich hätte ich den speziellen Fokus auf dem Traumabereich, um auch bereits bestehende Initiativen und Aktivitäten vernetzen zu helfen und zu unterstützen, z. B. durch Medienarbeit.

Gibt es ein Amt in der ASP, das Sie gerne bekleiden würden?

Ich bin sehr dankbar für jeden, der seine Zeit und Kräfte für die Arbeit des Verbandes gibt. Meinerseits sehe ich aktuell keine Kapazitäten für die Bekleidung eines Amtes in der ASP, obwohl mich Berufspolitik sehr interessiert und ich sie für eine sehr wichtige Arbeit halte.

Wie sähe Ihre Wunschsituation im gegebenen politischen Umfeld für PsychotherapeutInnen aus?

Psychotherapeuten und -therapeutinnen sollten als eigenständige Berufsgruppe anerkannt sein und ganz selbstständig wirken können. Damit verbunden sehe ich sowohl Rechte als auch Pflichten. Zu den Pflichten zähle ich auch, sich politisch und gesamtgesellschaftlich $\mathrm{zu}$ engagieren und das Wissen aktiv für die Gestaltung unseres Lebens und die Zukunft der nachfolgenden Generationen einzusetzen. Diese lassen wir mit gravierenden Problemen zurück, obwohl wir es besser wussten. Ich kann nachvollziehen, dass man seitens der Politik und Krankenkassen Angst vor einer Mengenausweitung bezüglich der psychotherapeutischen Leistung hat. Das stellt jedoch für mich einen gesamtgesellschaftlichen Konflikt dar, in dem die Ökonomisierung und ständiges Wachstum als oberstes Ziel stehen.

Was ist Ihre Vision in Ihrem beruflichen Alltag?

Ganz klar, dass der Psychotherapeutenberuf als selbstständige Berufsgruppe anerkannt wird, genauso wie der Psychiater eine anerkannte Berufsgruppe ist. Ich bin selbst Ärztin und schätze die Arbeit der psychiatrisch tätigen Kollegen und Kolleginnen sehr, wenn sie jedoch so umfassend Psychotherapie begreifen und verstehen wollten, wie PsychologInnen, bzw. Fachärzte für psychosomatische Medizin und Psychotherapie es tun, müssten sie zusätzlich eine Ausbildung von dem Umfang absolvieren und das Wissen kontinuierlich praktizieren, wie wir.

Dass dies auch vom gesellschaftlichen und politischen System so anerkannt wird. Auf Schweizer Formularen für Tagegeldversicherungen steht z. B. fettgedruckt, dass sie für die Einschätzung zwingend die Unterschrift eines Psychiaters fordern.

Dass man versteht, dass mehr Formalitäten und Dokumentationen, Qualitätssicherungsmassnahmen etc. eine enorme Ausbeutung menschlicher und hoch qualifizierter Ressourcen bedeuten und wir damit von unserer eigentlichen Arbeit abgehalten werden.

Meine Vision ist, dass Psychotherapeuten und Psychotherapeutinnen sich mit ihrem Wissen und ihren Fähigkeiten so stark wertschätzen, dass ihnen ihre Bedeutung im gesellschaftlichen Kontext klar bewusst wird. Ich glaube wirklich, dass Psychotherapeut bzw. Psychotherapeutin zu sein, eine Berufung ist.

Ich hoffe sehr, dass ich mit meinem sehr persönlichen Bericht andere Menschen zum Nachdenken anregen und vielleicht die eine oder andere Inspiration geben konnte.

Ich bedanke mich von ganzem Herzen für die Möglichkeit dieses Interviews!

\section{Zur Person}

Esther Bulang, Dr. med., ist eidgenössisch anerkannte Psychotherapeutin der ASP und Fachärztin für Augenheilkunde (FMH) sowie Fachärztin für Psychosomatische Medizin und Psychotherapie. Sie ist seit 2013 Mitglied der ASP.

Ihre Praxistätigkeit: Assistenzärztin und Psychotherapeutin bei Dr. med. Heidrun Kurz, Wotanstrasse 10, 8032 Zürich; sowie in eigener Praxis gemeinsam mit Frau Suna Baldinger, Psychologin SBAP, Limmatstrasse 107, 8005 Zürich estherbulang@gmail.com

Das Interview wurde schriftlich geführt von Veronica Defièbre. 